\title{
Bactericidal Activity of Mouse $\alpha$-Defensin Cryptdin-4 Predominantly Affects Noncommensal Bacteria
}

\author{
Koji Masuda ${ }^{a}$ Naoki Sakai ${ }^{a} b$ Kiminori Nakamura ${ }^{a, b}$ Sawako Yoshioka ${ }^{a}$ \\ Tokiyoshi Ayabe ${ }^{a, b}$ \\ a Innate Immunity Laboratory, Graduate School of Life Science, and ${ }^{b}$ Department of Cell Biological Science, \\ Faculty of Advanced Life Science, Hokkaido University, Sapporo, Japan
}

\section{Key Words}

Cryptdin-4 • Bacteria, commensal, noncommensal •

Disulfide bonds

\begin{abstract}
Mouse Paneth cell $\alpha$-defensins, termed cryptdins, are secreted into the intestinal lumen, have microbicidal activity, and contribute to intestinal innate immunity. Among them, cryptdin-4 (Crp4) has the most potent microbicidal activity. In the intestinal lumen, commensal bacteria colonize and elicit beneficial effects in the host. However, the effects of Crp4 against commensal bacteria are poorly understood. Thus, we investigated the bactericidal activities of Crp4 against commensal bacteria compared to noncommensal bacteria. Oxidized Crp4 showed only minimal or no bactericidal activity against 8 out of 12 commensal bacterial species, including Bifidobacterium bifidum and Lactobacillus casei. We further addressed a role of the conserved disulfide bonds of Crp4 by analyzing reduced Crp4 (r-Crp4). r-Crp4 demonstrated significantly greater bactericidal activities against 7 of 12 commensal bacteria than did oxidized Crp4. Oxidized Crp4 and r-Crp4 elicited equivalently potent bactericidal activities against 11 of the 11 noncommensal bacteria tested, such as Salmonella enterica serovar Typhimurium,
\end{abstract}

and against 5 of 12 commensal bacteria. Furthermore, when r-Crp4 was exposed to a processing enzyme of cryptdins, i.e. MMP-7, r-Crp4 was degraded and the bactericidal activities disappeared. These findings suggest that $\mathrm{Crp} 4$ has selective bactericidal activities against intestinal microbiota and that the activities are dependent on the disulfide bonds.

Copyright $\odot 2010$ S. Karger AG, Basel

\section{Introduction}

Innate immunity functions as the front line of host defense in plants, invertebrates, and mammals. Antimicrobial peptides (AMPs) are one of the major effectors of innate immunity [1-3]. In the small intestine, AMP $\alpha$ defensins are expressed in the granules of Paneth cells and are secreted into the lumen of intestinal crypts in response to bacterial stimuli [4]. The secreted $\alpha$-defensins elicit potent bactericidal activity and contribute to innate immunity in the small intestine $[5,6]$. Mouse $\alpha$-defensins, termed cryptdins (Crps), are activated in vivo in $\mathrm{Pa}$ neth cell granules through the processing of pro-Crps with the proteolytic enzyme matrix metalloproteinase-7 (MMP-7; matrilysin) [7, 8]. MMP-7 cleaves pro-Crps at 3 cleavage sites, and 1 of these sites is the $\mathrm{N}$-terminus or

\section{KARGER}

Fax +4161306 1234

E-Mail karger@karger.ch

www.karger.com (c) 2010 S. Karger AG, Basel

1662-811X/11/0033-0315\$38.00/0

Accessible online at:

www.karger.com/jin
Prof. Tokiyoshi Ayabe

Department of Cell Biological Science

Graduate School of Life Science, Hokkaido University

N21, W11, Kita-ku, Sapporo, Hokkaido 001-0021 (Japan)

Tel. +81 11706 9049, Fax +81 11706 9053, E-Mail ayabe@ sci.hokudai.ac.jp 
near the $\mathrm{N}$-terminus of mature Crps [9]. This processing is an essential event for the production of mature, functional Crps [7-9]. MMP-7-deficient mice that lack the active form of Crps are significantly more susceptible to orally administered Salmonella enterica serovar Typhimurium than are wild-type mice [7]. These results also show the pivotal role of AMPs in innate immunity.

In the gastrointestinal tract, a highly complex microbial ecosystem is constructed by colonizing microbes. Hosts and microbiota have coevolved in ways that have mutually beneficial effects [10]. These include host development [11], nutritional absorption [12], and the functional development of the immune systems [13]. Recent studies have shown that well-balanced cross talk between the host and commensal bacteria is important [14] as imbalances in the relationships result in inflammation [15] and cancer [16]. In the mouse small intestine, the expression of more than 20 cryptdin genes and cryptdin-related sequence genes has been reported [17-20], and these peptides are secreted into the intestinal lumen where commensal bacteria reside [21]. Among Crps, cryptdin-1 to cryptidin-6 (Crp1 to Crp6) are characterized at the peptide level. Although the amino acid sequence identities of mature regions of $\mathrm{Crp} 1$ to $\mathrm{Crp} 3$ and Crp6 are more than 90\%, those of Crp4 and Crp5 with Crp1 are 42 and 54\%, respectively [18]. In particular, Crp4 has several features that distinguish it from other Crps. For example, the Crp4 polypeptide chain uniquely lacks 3 amino acids between the fourth and fifth cysteine residue positions [18] and the unique repeated element in the region upstream of the gene transcriptional start site [22]. Crp4 also has the most potent in vitro bactericidal activity of known mouse Paneth cell $\alpha$-defensins [18], suggesting that Crp4 may play a pivotal role in intestinal innate immunity. However, the interaction of Crp4 and small intestinal microbiota is poorly understood. Thus, to clarify the effects of Crp4 on commensal bacteria, we investigated the bactericidal activities of Crp4 against commensal bacteria compared to noncommensal bacteria. We further tested the bactericidal activities of Crpl in addition to Crp4.

Crp4 is a highly cationic peptide and is generally believed to permeabilize the bacterial plasma membrane through electrostatic interaction with negatively charged bacterial phospholipids followed by the insertion of hydrophobic side chains [23]. This conclusion is supported by reports which reveal that positively charged Arg residues of Crp4 are critical for its bactericidal activity [24]. However, the precise mechanisms of its bactericidal activity are not known. $\alpha$-Defensins including Crp 4 are characterized by invariant disulfide bonds arranged be- tween $\mathrm{Cys}^{1}-\mathrm{Cys}^{6}$, $\mathrm{Cys}^{2}-\mathrm{Cys}^{4}$, and $\mathrm{Cys}^{3}-\mathrm{Cys}^{5}$ [3]. The pairings of 3 disulfide bonds are conserved in all species which express these peptides. Previously, the bactericidal activities of the Crp4 mutants in which Cys residues were substituted to Ala residues were analyzed by Maemoto et al. [25], who showed that disulfide bond-null mutants had equivalent or greater bactericidal activity compared to native Crp4 [25]. However, the effects of the disulfide bonds in native $\mathrm{Crp} 4$ on the bactericidal activity against commensal bacteria remain unknown. Therefore, we further addressed a role of the disulfide bonds in the bactericidal activity of Crp4 using reduced Crp4 (r-Crp4) which did not contain disulfide bonds.

Crp4 is processed by MMP-7 in the granules of Paneth cells. It has been reported that the disulfide bond-null mutant of Crp4 is degraded by MMP-7 [25]. This result indicates that the disulfide bonds of Crp4 determine the proteolytic resistance to MMP-7. Therefore, to elucidate the effect of reduction on the processing of $\mathrm{r}$-Crp4, we also investigated the susceptibility of r-Crp4 to MMP-7 and the effects of MMP-7 on the bactericidal activity of the processed $\mathrm{r}-\mathrm{Crp} 4$.

\section{Materials and Methods}

Preparation of Oxidized Crps and r-Crps

Three pairs of disulfide bonds were introduced into Crp4 (Sigma Genosys, St. Louis, Mo., USA, or Medical and Biological Laboratories Co., Ltd., Nagoya, Japan) by air oxidation as described previously [25]. Crp4 was then purified to homogeneity using reverse-phase high-performance liquid chromatography (RPHPLC). The Crp4 with 3 pairs of disulfide bonds, defined as oxidized Crp4, was purified by a C-18 column (SepaxHP-C18, $4.6 \times$ 150 mm, $5 \mu \mathrm{m}$; Sepax Technologies, Inc., Newark, Del., USA) in $0.1 \%$ trifluoroacetic acid with an $18-36 \%$ acetonitrile gradient developed over $30 \mathrm{~min}$ at $1 \mathrm{ml} / \mathrm{min}$ (online supplementary figure S1a, www.karger.com/doi/10.1159/000322037). Oxidized Crp4 was obtained after the final lyophilization and stored at $-30^{\circ} \mathrm{C}$ until use.

r-Crp4, in which the disulfide bonds were entirely reduced, was prepared. Oxidized Crp4 was dissolved in $500 \mathrm{~mm}$ dithiothreitol (DTT) and left to stand at $4{ }^{\circ} \mathrm{C}$ overnight. The reaction mixture was then applied to a C-18 column and $\mathrm{r}$-Crp4 was purified by RP-HPLC under the same conditions used in the purification of oxidized Crp4 (online suppl. fig. S1b). Because the retention times of DTT and r-Crp4 were very different, we concluded that DTT was completely removed from r-Crp4 in the purification process. Thus, the r-Crp4 used in the assay did not contain DTT, and we confirmed that DTT did not affect the bactericidal assay. r-Crp4 was obtained after the final lyophilization and stored at $-30^{\circ} \mathrm{C}$ until use. Oxidized $\mathrm{Crpl}$ and r-Crp1 were also prepared by the same respective methods. 
Evaluation of Disulfide Bond Formation

Disulfide bond formation was evaluated by acid-urea PAGE (AU-PAGE) [26], MALDI-TOF MS, and RP-HPLC. In the AUPAGE analysis, samples $(1.0 \mu \mathrm{g})$ of oxidized Crp4 and r-Crp4 were dissolved in 5\% acetic acid and electrophoresed on $12.5 \%$ acrylamide gel containing 5\% acetic acid and $5 \mathrm{M}$ urea at $150 \mathrm{~V}$ [26]. Thereafter, the gel was stained with Coomassie brilliant blue R-250. The molecular masses of the peptides were determined by MALDI-TOF MS (Voyager-DE PRO; Applied Biosystems, Carlsbad, Calif., USA). Oxidized Crp4 and r-Crp4 were analyzed by RP-HPLC using a C-18 column under the same conditions used in the purification of oxidized Crp4.

\section{Bacterial Strains and Culture Conditions}

As some of the most common commensal bacteria in the small intestine [27-30], Bifidobacterium bifidum ATCC 11863 (B. bifidum), Bifidobacterium breve JCM 1192 (B. breve), Bifidobacterium longum ATCC 15707 (B. longum), Lactobacillus acidophilus ATCC 314 (L. acidophilus), Lactobacillus casei ATCC 393 (L. casei), Lactobacillus johnsonii JCM 2012 (L. johnsonii), Bacteroides fragilis JCM 11019 (B. fragilis), Bacteroides ovatus JCM 5824 (B. ovatus), Bacteroides thetaiotaomicron JCM 5827 (B. thetaiotaomicron), Bacteroides vulgatus JCM 5826 (B. vulgatus), Enterococcus faecalis JCM 5803 (E. faecalis), and Enterococcus faecium JCM 5804 (E. faecium) were used. As examples of noncommensal bacteria in the small intestine, wild-type $S$. enterica serovar Typhimurium ATCC 14028 (S. enterica serovar Typhimurium), a defensin-sensitive strain of $S$. enterica serovar Typhimurium phoP- (S. enterica serovar Typhimurium phoP-) [31], Escherichia coli ML35 ATCC 43827 (E. coli), Staphylococcus aureus ATCC 27217 (S. aureus), Listeria monocytogenes JCM 7671 (L. monocytogenes), Klebsiella oxytoca JCM 1665 (K. oxytoca), Klebsiella pneumoniae JCM 1662 (K. pneumoniae), Proteus vulgaris JCM 20013 (P. vulgaris), Yersinia enterocolitica JCM 7577 (Y. enterocolitica), Campylobacter coli JCM 2529 (C. coli), and Campylobacter jejuni JCM 2013 (C. jejuni) were used. Bacteria were cultured in the following media: $B$. bifidum, reinforced clostridial medium (RCM) supplemented with $2 \%$ (w/v) skim milk; B. breve and B. longum, RCM; Lactobacillus sp., de Man, Rogosa, and Sharpe (MRS) broth; Bacteroides sp., GAM broth (Nissui Seiyaku Co., Ltd., Tokyo, Japan); Enterococcus sp., brain heart infusion (BHI); noncommensal bacteria except for C. coli and C. jejuni, tryptic soy broth, and C. coli and C. jejuni, GAM broth. Commensal bacteria were grown in anaerobic conditions using the Anaero Pack system (Mitsubishi Gas Chemical Co., Inc., Tokyo, Japan) at $37^{\circ} \mathrm{C}$. Noncommensal bacteria, except for $C$. coli and C. jejuni, were grown in a shaking incubator at $37^{\circ} \mathrm{C}$ with shaking at $180 \mathrm{rpm}$. C. coli and C. jejuni were grown in microaerophilic conditions using the Anaero Pack system.

\section{Bactericidal Peptide Assay}

Exponential-phase bacteria cultured at $37^{\circ} \mathrm{C}$ were deposited by centrifugation at $9,300 \mathrm{~g}$ at $4^{\circ} \mathrm{C}$ for $5 \mathrm{~min}$. Bacteria, except for $B$. vulgatus, were washed twice and resuspended in Milli-Q water; $B$. vulgatus was washed twice and resuspended in PBS diluted 1:4 with Milli-Q water. The $\mathrm{OD}_{620}$ was measured to determine bacterial cell numbers. Twenty microliters of samples containing 1,000 colony-forming units (CFU) per aliquot were mixed with equal volumes of oxidized Crp4, r-Crp4, oxidized Crp1, or r-Crp1 to final concentrations ranging from 0.027 to $1.35 \mu \mathrm{M}$. The mixtures were incubated for $1 \mathrm{~h}$ at $37^{\circ} \mathrm{C}$. The incubated samples were plated on RCM agar plates for Bifidobacterium sp., on MRS agar plates for Lactobacillus sp., on GAM agar plates for Bacteroides sp., on BHI agar plates for Enterococcus sp., and on tryptic soy agar (TSA) plates for noncommensal bacteria. The plates were then incubated in anaerobic conditions at $37^{\circ} \mathrm{C}$ for commensal bacteria or at $37^{\circ} \mathrm{C}$ for noncommensal bacteria. Bacterial survival rates were determined from surviving colonies relative to peptide-unexposed controls (online suppl. fig. S2). The bacterial cell viability of the peptide-unexposed controls was not changed during bactericidal peptide assay (data not shown).

\section{Bactericidal Peptide Assay against a Bacterial Mixture}

Exponential phase S. enterica serovar Typhimurium, L. casei, and $B$. thetaiotaomicron cultured at $37^{\circ} \mathrm{C}$ were washed with Milli-Q water, and each bacteria population was adjusted to $150 \mathrm{CFU}$ in $20 \mu \mathrm{l}$. Subsequently, each bacterial solution of $20 \mu \mathrm{l}$ was mixed, and the mixture was incubated with $60 \mu$ l of oxidized Crp4 or rCrp4 with a final concentration of $1.35 \mu \mathrm{M}$. After incubation for $1 \mathrm{~h}$ at $37^{\circ} \mathrm{C}$, the bacterial mixtures were separated and grown on TSA plates in aerobic conditions and on MRS and GAM plates in anaerobic conditions using the Anaero Pack system. More than 28 colonies were picked randomly from each plate as being representative of the total colonies. Subsequently, the genomic sequences of the conserved region in 16S rRNA among 3 species of bacteria were amplified by colony-direct polymerase chain reaction (PCR) using Blend Taq (TOYOBO, Tokyo, Japan). The forward primer (5'-GTTGG TGAGG TAACG GCTCA CCAA-3') was paired with the reverse primer ( $5^{\prime}$-TGACG GGCGG TGTGT ACAAG GC-3'). The PCR products from S. enterica serovar Typhimurium, $L$. casei, and $B$. thetaiotaomicron were digested at only 1 site by BamH I, $B g l$ II, and Spe I, respectively. Therefore, after the digestion of the PCR products by these 3 enzymes, each bacterium was distinguished by the resulting patterns visualized by agarose electrophoresis. The relative bacterial distribution was shown based on chosen representative colonies, and the actual colony numbers of each bacterium were estimated by multiplying the relative bacterial distribution by the total colony numbers. Because each plate is appropriate for each bacterium, the numbers of S. enterica serovar Typhimurium, L. casei, and B. thetaiotaomicron were estimated from colonies grown on TSA, MRS, and GAM plates, respectively.

Antimicrobial Assay with Membrane Potential-Sensitive Dye

Exponential-phase bacteria were incubated in Milli-Q water at $37^{\circ} \mathrm{C}$ with oxidized Crp4 or r-Crp4 $(1.35 \mu \mathrm{M})$ for $1 \mathrm{~h}$. The suspensions were then incubated for $10 \mathrm{~min}$ with $1 \mu \mathrm{g} / \mathrm{ml}$ of the membrane potential-sensitive fluorophore, i.e. bis-(1,3-dibutylbarbituric acid) trimethine oxonol $\left[\mathrm{DiBAC}_{4}(3)\right]$ (Invitrogen, Carlsbad, Calif., USA), as described previously [32]. The suspensions were centrifuged for $5 \mathrm{~min}$ at $9,300 \mathrm{~g}$, and the bacterial pellets were resuspended in $1 \mathrm{ml}$ PBS(-). Each bacterial sample was analyzed on a JSAN desktop cell sorter (Bay Bioscience, Kobe, Japan). The median fluorescence intensity ratio was obtained by dividing the median fluorescence intensity of the peptide-treated sample by the median fluorescence intensity of the nontreated sample. 
Cleavage of $r$-Crp4 with MMP-7 in vitro

Samples $(1.0 \mu \mathrm{g})$ of oxidized Crp4 and r-Crp4 were incubated with an activated recombinant human MMP-7 (1.0 $\mu \mathrm{g})$ catalytic domain (Calbiochem, La Jolla, Calif., USA) in $10 \mathrm{~mm}$ HEPES $\mathrm{pH} 7.4,150 \mathrm{mM} \mathrm{NaCl}$, and $5 \mathrm{mM} \mathrm{CaCl}_{2}$ for $18-24 \mathrm{~h}$ at $37^{\circ} \mathrm{C}[9,25]$. The digested samples were analyzed by Tris-Tricine SDS-PAGE, N-terminal peptide sequencing, and MALDI-TOF MS. For the $\mathrm{N}$-terminal peptide sequencing by Edman degradation, digested r-Crp 4 was resolved by RP-HPLC under the same conditions used

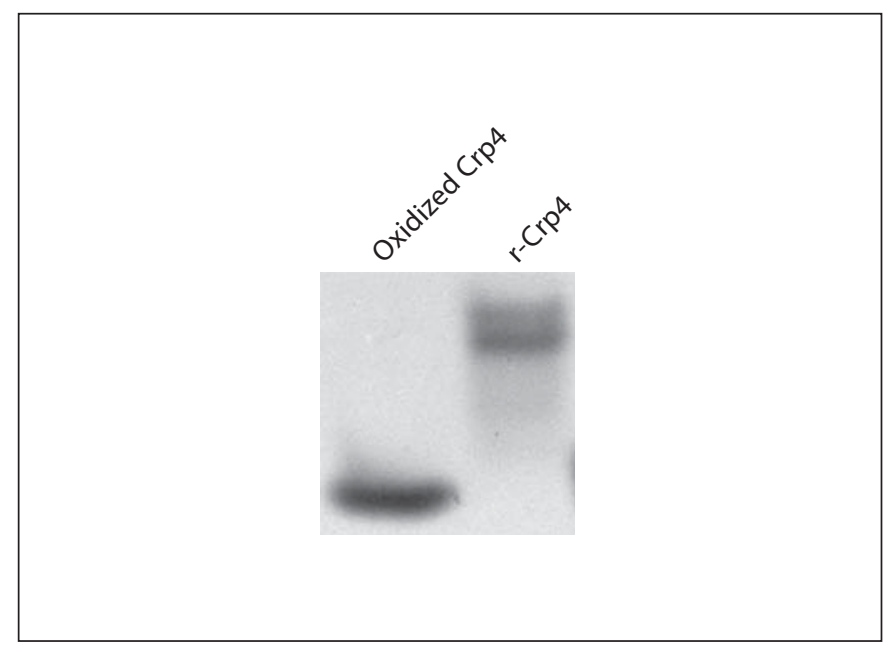

Fig. 1. AU-PAGE analysis of oxidized Crp4 and r-Crp4. Samples $(1.0 \mu \mathrm{g})$ of oxidized Crp4 and r-Crp4 were resolved by AU-PAGE (12.5\% acrylamide gel containing $5 \%$ acetic acid and $5 \mathrm{M}$ urea) and then stained with Coomassie brilliant blue R-250. in the purification of oxidized Crp4. Samples (50 pmol) of digested r-Crp4 were subjected to 5 cycles of $\mathrm{N}$-terminal peptide sequencing at the Creative Research Initiative 'Sousei', Hokkaido University. To determine biological activity, the digested samples were applied to bactericidal peptide assays as described above.

\section{Results}

Oxidized Crp4 Shows Bactericidal Activities against Noncommensal Bacteria but Little or No Bactericidal Activity against Some Commensal Bacteria

Throughout the present study, we used oxidized Crp4 containing 3 pairs of disulfide bonds and r-Crp4 obtained by the reduction of oxidized Crp4. As shown in the results of AU-PAGE (fig. 1), MALDI-TOF MS (fig. 2a), and RPHPLC (online suppl. fig. S1a), oxidized Crp4 was homogeneous. Oxidized Crp4 showed a lower molecular weight than $\mathrm{r}$-Crp 4 by $6 \mathrm{Da}$ (fig. 2), consistent with the oxidation of 6 Cys residues in the formation of disulfide pairings. Furthermore, oxidized Crp4 had bactericidal activity against 2 strains of $S$. enterica serovar Typhimurium, as well as against $E$. coli and S. aureus (fig. 3). Hence, the prepared oxidized Crp4 was biologically active and contained 3 pairs of disulfide bonds. $r$-Crp4 showed a reduced migration relative to oxidized Crp4 in AU-PAGE (fig. 1). This corresponds to the results of AU-PAGE for oxidized Crp4 versus Cys-to-Ala-substituted Crp4, in which the disulfide-null mutant showed a reduced migration compared to oxidized Crp4 [25]. MALDI-TOF MS of

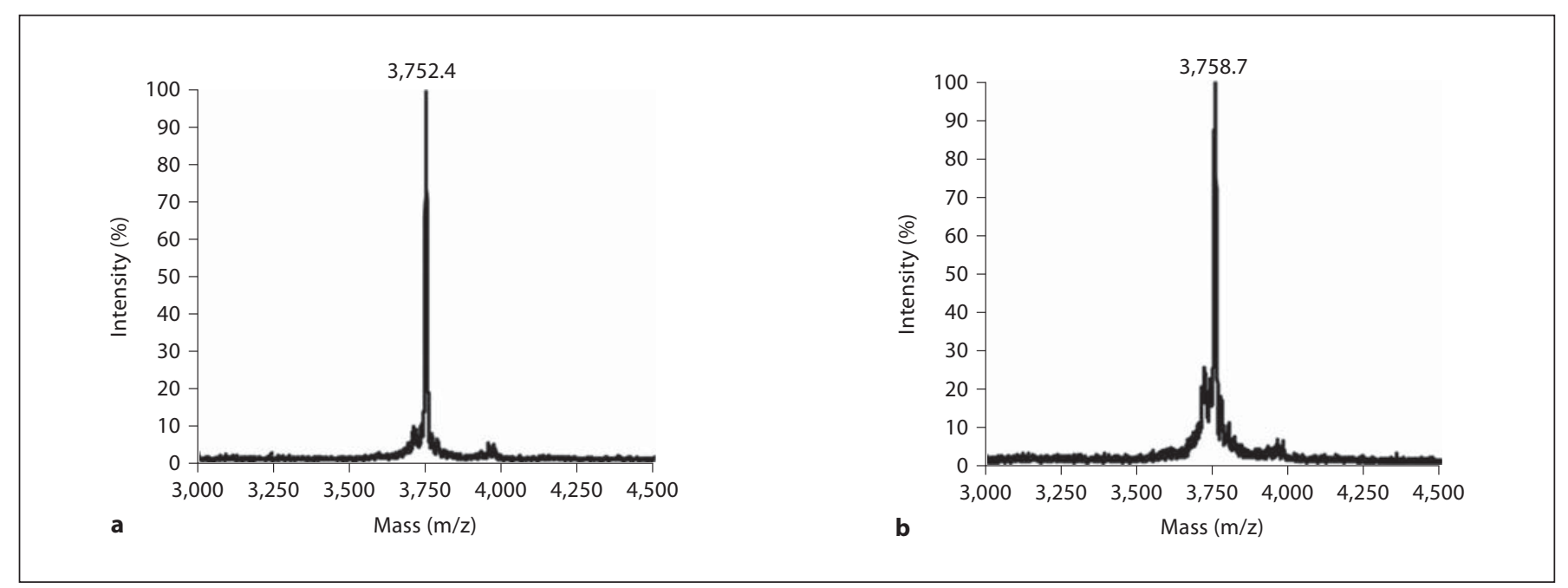

Fig. 2. MALDI-TOF MS analysis of oxidized Crp4 and r-Crp4. The molecular weights of oxidized Crp4 (a) and r-Crp4 (b) analyzed by MALDI-TOF MS are shown. 
Fig. 3. Bactericidal activities of oxidized Crp4 and r-Crp4 against noncommensal bacteria. The survival rates of $S$. enterica serovar Typhimurium (a), S. enterica serovar Typhimurium phoP- (b), E. coli ML35 (c), S. aureus (d), L. monocytogenes (e), K. oxytoca (f), K. pneumoniae (g), P. vulgaris (h), Y. enterocolitica (i), C. coli (j), and $C$. jejuni (k) exposed to oxidized Crp4 $(\bullet)$ or r-Crp4 $(\square)$ at $0.027,0.054,0.135$, 0.27 , and $1.35 \mu \mathrm{M}$ are shown. Data are expressed as means $\pm \mathrm{SE} ; \mathrm{n}=6$ for $\mathbf{a}-\mathbf{d}$; $\mathrm{n}=$ 3 for e-k. Experiments were performed in triplicate.

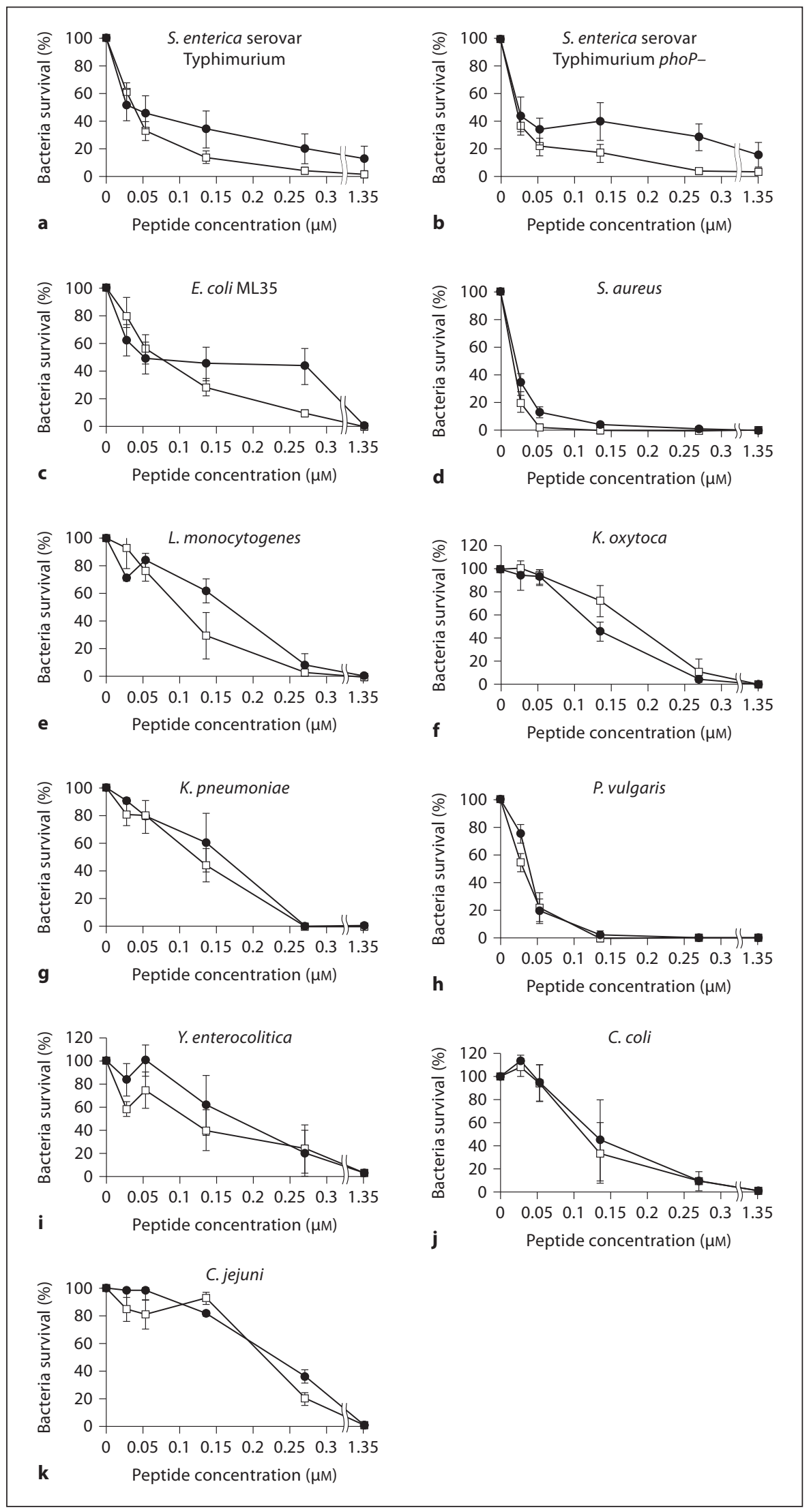


Fig. 4. Bactericidal activities of oxidized Crp4 and r-Crp4 against commensal bacteria. The survival rates of B. bifidum (a), B. breve (b), B. longum (c), L. acidophilus (d), L. casei (e), L. johnsonii (f), B. fragilis (g), B. ovatus (h), B. thetaiotaomicron (i), $B$. vulgatus (j), E. faecalis (k), and E. faecium (I) exposed to oxidized Crp4 (•) or r-Crp4 $(\square)$ at $0.027,0.054,0.135,0.27$, and $1.35 \mu \mathrm{M}$ are shown. Data are expressed as means \pm $\mathrm{SE} ; \mathrm{n}=6$ for $\mathbf{a}, \mathbf{c}-\mathbf{e}, \mathbf{i}$, and $\mathbf{j} ; \mathrm{n}=3$ for $\mathbf{b}, \mathbf{f}-\mathbf{h}$, $\mathbf{k}$, and $\mathbf{I}$. Experiments were performed in triplicate. ${ }^{*} \mathrm{p}<0.01$ by Student's t test.
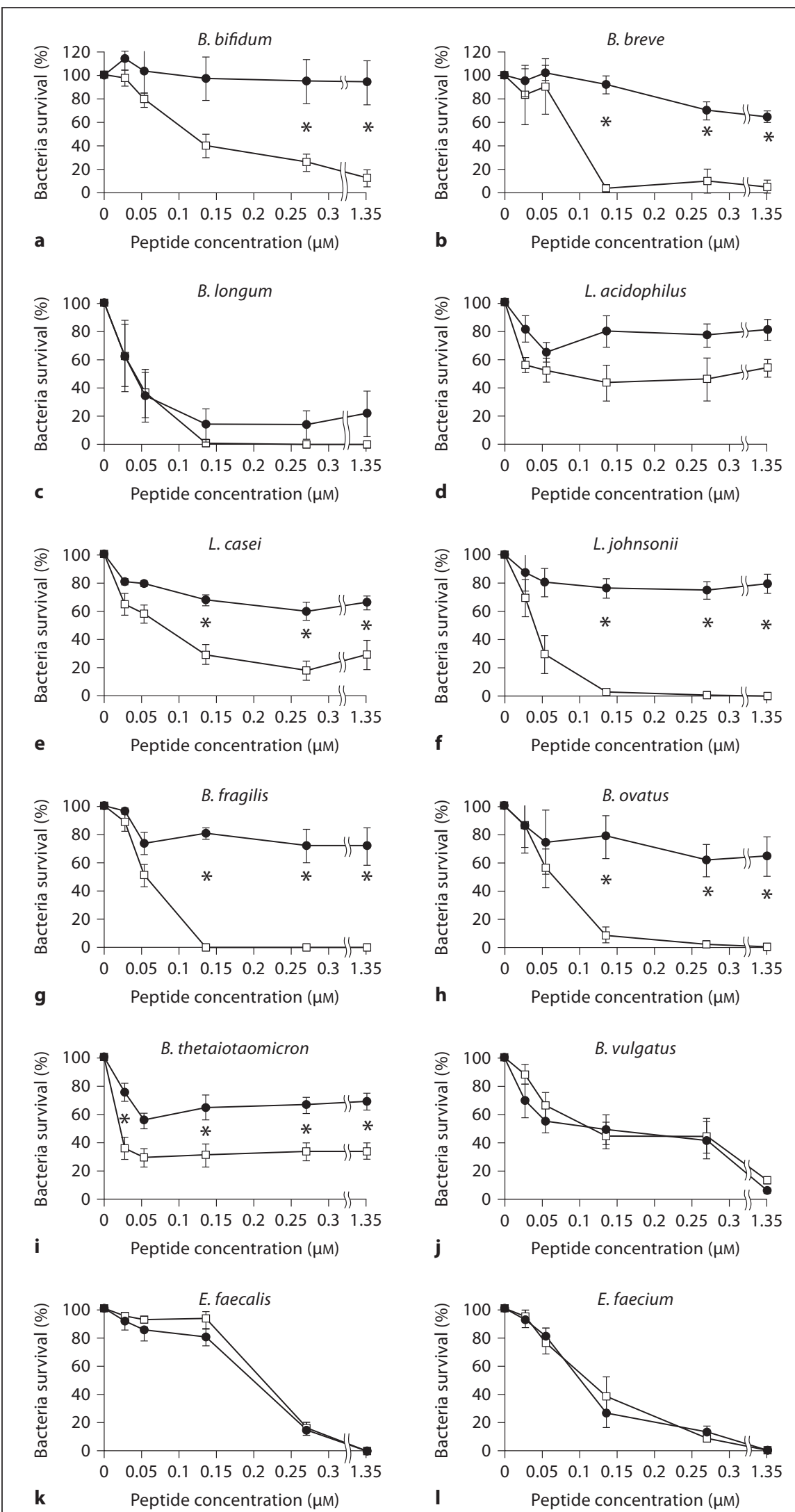


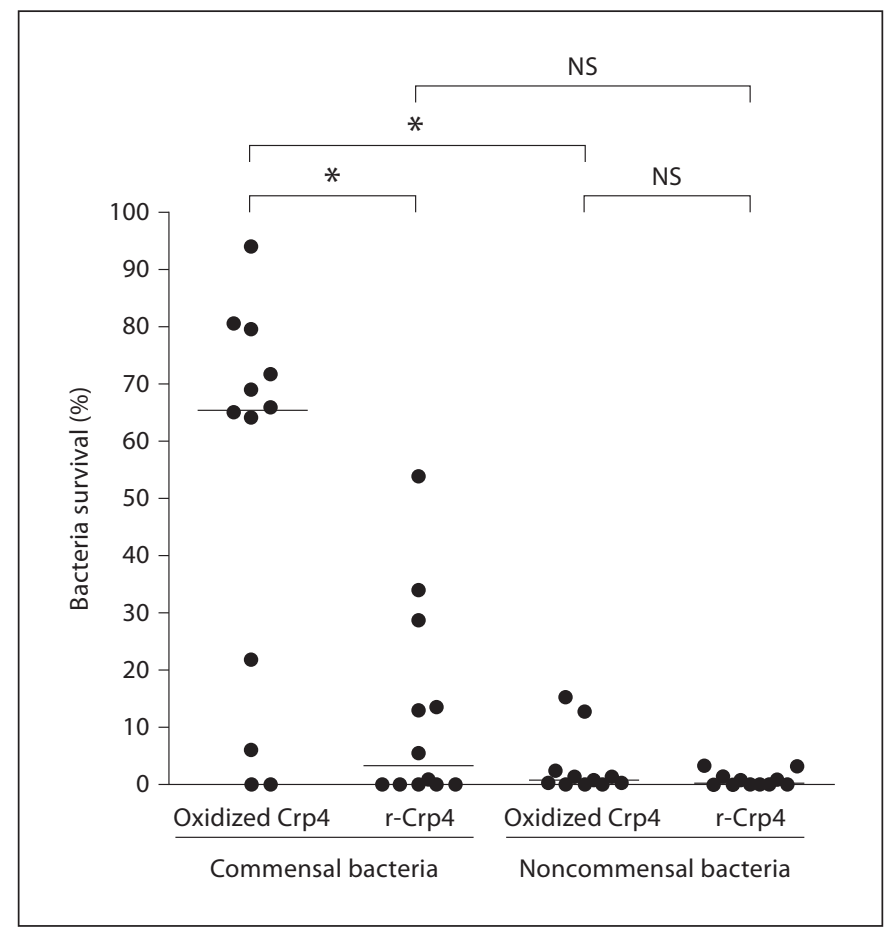

Fig. 5. Statistical analysis of bactericidal activities. The survival rates of 12 commensal bacteria and 11 noncommensal bacteria (Material and Methods) exposed to oxidized Crp4 or r-Crp4 at $1.35 \mu \mathrm{M}$ are shown. Each point represents a survival rate of each bacterium. The medians are expressed as horizontal lines; the data were evaluated for statistical significance using the MannWhitney $U$ test where differences at values of ${ }^{*} p<0.05$ were considered to be significant. NS = Not significant.

r-Crp4 showed a major peak (fig. 2b) and RP-HPLC of rCrp4 showed a single peak (online suppl. fig. S1b). From these results, we judged that r-Crp4 was homogeneous and that the 3 pairs of disulfide bonds were reduced. The reduction state of $\mathrm{r}$-Crp4 was assured by MALDI-TOF MS and AU-PAGE prior to use and after incubation in bactericidal assays. Although r-Crp4 showed secondary bands in AU-PAGE suggestive of disulfide bond formation, some Cys residues may tend to form disulfide bonds in AUPAGE since an excess concentration of DTT is needed for the complete reduction of Crp4.

The bactericidal activities of oxidized Crp4 against small intestinal commensal and noncommensal bacteria were examined. Oxidized Crp4 killed 11 of 11 noncommensal bacteria and 4 of 12 commensal bacteria, i.e. B. longum, B. vulgatus, E. faecalis, and E. faecium, in a dose-dependent manner (fig. 3, 4c, j-1). In contrast, oxidized Crp4 showed little or no bactericidal activities against 8 out of 12 commensal bacteria, i.e. B. bifidum,

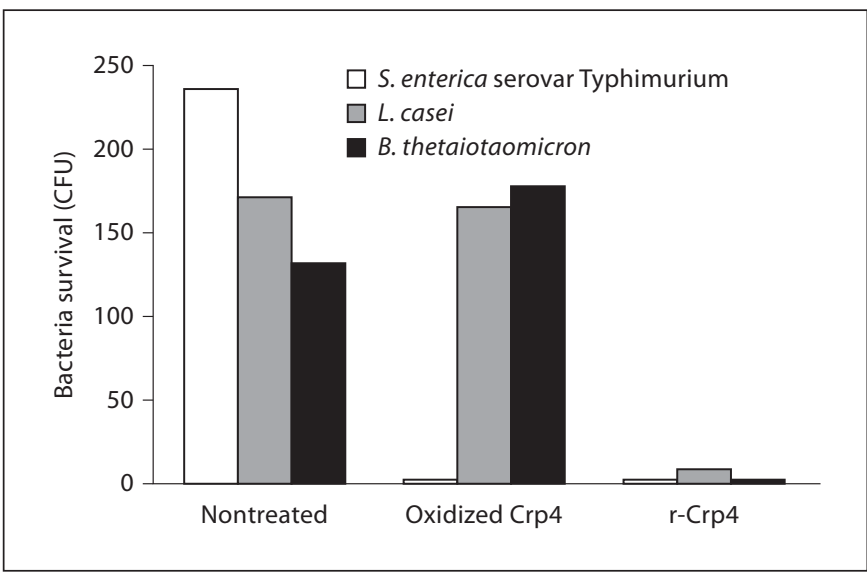

Fig. 6. Bactericidal activities of oxidized Crp4 and r-Crp4 against the mixture of commensal and noncommensal bacteria. The bacterial mixture of exponentially growing $S$. enterica serovar Typhimurium as noncommensal bacteria and $L$. casei and $B$. thetaiotaomicron as commensal bacteria was exposed to oxidized Crp4 or r-Crp4 at $1.35 \mu \mathrm{M}$. The surviving bacteria were counted as CFU.

B. breve, L. acidophilus, L. casei, L. johnsonii, B. fragilis, B. ovatus, and B. thetaiotaomicron at $1.35 \mu \mathrm{M}$ (fig. $4 \mathrm{a}, \mathrm{b}$, $\mathrm{d}-\mathrm{i})$. These activities did not change at a $2.7 \mu \mathrm{M}$ peptide concentration (data not shown). Thus, oxidized Crp4 had only a minimal effect or no effect on the survival of 8 out of the 12 species of commensal bacteria tested. In contrast, oxidized Crp4 had potent bactericidal activities against 11 of the 11 noncommensal bacterial species tested, but it was only active against 4 of the 12 species of commensal bacteria. The statistical analysis of the bactericidal activities of oxidized Crp4 against commensal bacteria and noncommensal bacteria revealed that oxidized Crp4 showed significantly greater bactericidal activities against noncommensal bacteria than against commensals at $1.35 \mu \mathrm{M}$ (fig. 5; commensal bacteria vs. noncommensal bacteria exposed to oxidized Crp4).

In the intestinal lumen, a wide variety of bacteria are able to colonize. To further test the selective activity of oxidized Crp4, a mixture of commensal and noncommensal bacteria was exposed to oxidized Crp4. Oxidized Crp4 selectively killed S. enterica serovar Typhimurium, while the viability of the other commensal bacteria, i.e. $L$. case $i$ and B. thetaiotaomicron, in the mixture was retained (fig. 6; nontreated and oxidized Crp4-treated). This result supports the selective activities of Crp4.

To determine whether this selective activity is specific to oxidized Crp4, we analyzed the bactericidal activities of oxidized Crp1, the most abundant Crp, against 6 non- 


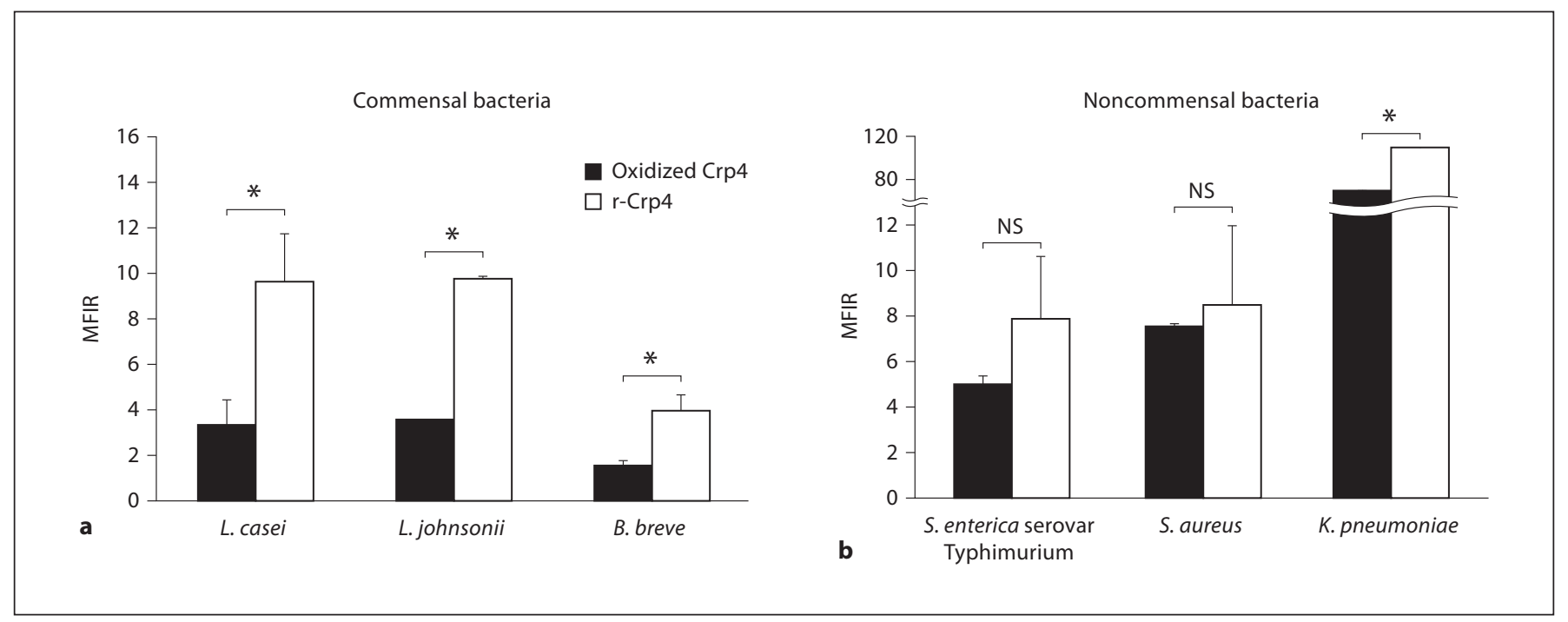

Fig. 7. Antimicrobial assay with membrane-sensitive dye. The median fluorescence intensity ratios (MFIR) of commensal bacteria (a) and noncommensal bacteria (b) exposed to oxidized Crp4 ( $\mathbf{a})$ or r-Crp4 ( $\square$ ) are shown. Data are expressed as means \pm SE. Experiments were performed in triplicate, and the results were evaluated for statistical significance by Student's t test. ${ }^{*} \mathrm{p}<0.05$ was considered to be statistically significant. NS $=$ Not significant.

commensal bacteria, i.e. S. enterica serovar Typhimurium, S. aureus, E. coli, L. monocytogenes, K. oxytoca, and $P$. vulgaris, as well as against 6 commensal bacteria, i.e. B. bifidum, B. longum, L. casei, L. johnsonii, B. fragilis, and $B$. thetaiotaomicron. Oxidized Crp1 showed potent bactericidal activities against 6 of 6 noncommensal bacteria and against 1 of 6 commensal bacteria, i.e. B. longum (online suppl. table S1). However, Crp1 had little or no effect on 5 of 6 commensal bacteria (online suppl. table S1). Thus, oxidized Crpl also had predominant bactericidal activities against noncommensal bacteria, as did oxidized Crp4.

\section{$r$-Crp4 Has Bactericidal Activities against both Commensal and Noncommensal Bacteria}

Since disulfide bond pairings in $\alpha$-defensins are conserved in all species which express these peptides, the bactericidal activities of $\mathrm{r}$-Crp4 were analyzed to elucidate the effects of the conserved disulfide bonds on its bactericidal activity. $r$-Crp4 showed equivalent bactericidal activities against all noncommensal bacteria but only against 5 of 12 commensal bacterial species, i.e. $B$. longum, L. acidophilus, B. vulgatus, E. faecalis, and E. faecium, in comparison with oxidized Crp4 (fig. 3, 4c, d, j-l). These data indicated that the bactericidal activities of Crp4 against these bacteria were independent of the ex- istence of the disulfide bonds. On the other hand, r-Crp4 killed 7 out of 12 commensal bacteria, i.e. B. bifidum, $B$. breve, $L$. casei, L. johnsonii, B. fragilis, B. ovatus, and B. thetaiotaomicron, in a dose-dependent manner and showed significantly greater bactericidal activities compared to those of oxidized Crp4 (fig. 4a, b, e-i). Therefore, the reduction of the disulfide bonds conferred Crp4 bactericidal activities against $B$. bifidum, B. breve, L. casei, $L$. johnsonii, B. fragilis, B. ovatus, and B. thetaiotaomicron, but the disulfide bonds had little or no effect on bactericidal activity against all noncommensal bacteria tested and B. longum, L. acidophilus, B. vulgatus, E. faecalis, and E. faecium. A comparison of the bactericidal activities of oxidized Crp4 and r-Crp4 revealed that r-Crp4 had significantly greater bactericidal activities against commensal bacteria than did oxidized Crp4 (fig. 5; commensal bacteria exposed to oxidized Crp4 vs. r-Crp4). The bactericidal activities of oxidized Crp4 and r-Crp4 were not significantly different against noncommensal bacteria (fig. 5; noncommensal bacteria exposed to oxidized Crp4 vs. $r$-Crp4).

When the mixture of commensal and noncommensal bacteria was exposed to r-Crp4, r-Crp4 had potent bactericidal activities against both commensals and noncommensals (fig. 6; nontreated and r-Crp4-treated). Therefore, the selective bactericidal activities of Crp4 


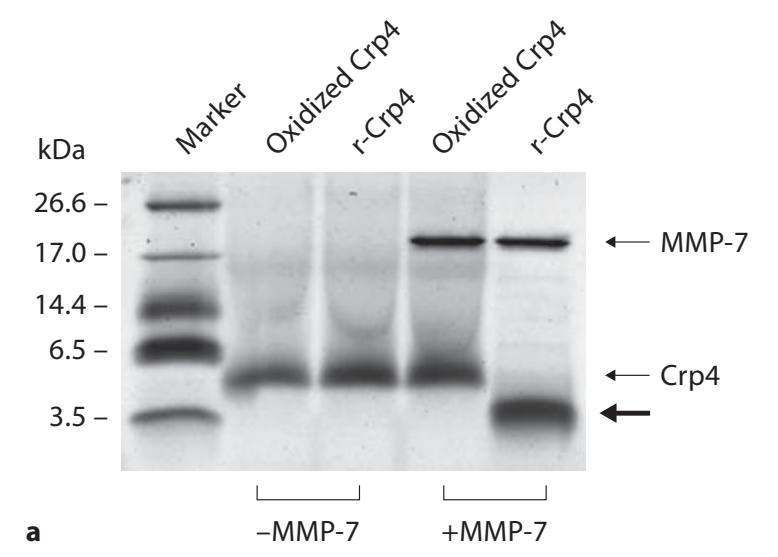

b

Fig. 8. Proteolytic degradation assay of oxidized Crp4 and r-Crp4 by MMP-7. a Tris-Tricine SDS-PAGE analysis of oxidized Crp4 and $\mathrm{r}-\mathrm{Crp} 4$ incubated with or without MMP-7. Samples $(1.0 \mu \mathrm{g})$ of oxidized Crp4 and r-Crp4 were incubated with or without an MMP-7 (1.0 $\mu \mathrm{g})$ catalytic domain. The digested samples were analyzed by Tris-Tricine SDS-PAGE. The positions of bands corresponding to MMP-7 and Crp4 are noted on the right. The bold arrow denotes the position of the MMP-7-digested fragment of r-Crp4. b MMP-7 cleavage sites in r-Crp4. The digested r-Crp4 was analyzed by 5 cycles of $\mathrm{N}$-terminal peptide sequencing and MALDI-TOF MS. Cleavage sites are noted by downward arrows $(\downarrow)$. The numerals below the r-Crp4 sequence refer to residue positions in reference to the $\mathrm{N}$-terminal Gly of $\mathrm{r}$-Crp4 as residue position number 1 .

against certain commensal bacteria were regulated by its disulfide bonds.

Furthermore, the bactericidal activities of r-Crp1 against 6 noncommensal and 6 commensal bacteria were also tested for relative sensitivity to a second mouse $\alpha$ defensin, oxidized Crp1 and r-Crp1. r-Crp1 showed bactericidal activities equivalent to those of oxidized Crp1 against 6 of 6 noncommensal bacteria and against 1 of 6 commensal bacteria, i.e. B. longum (online suppl. table S1), but it demonstrated significantly greater bactericidal activity against 5 of 6 commensal bacteria (online suppl. table S1) compared to oxidized Crp1. Thus, the predominant bactericidal activities of Crpl against noncommensal bacteria were also affected by its disulfide bonds, as in the case of Crp4.

We next tested the membrane disruption activity of Crp4. It has been reported that a Crp4-permeabilized

bacterial cell membrane and permeabilization correlate with bacterial killing activity [23]. To examine the possible mechanism of the bactericidal activities of Crp4, a depolarization of the membrane potential was detected with membrane potential-sensitive fluoroprobe Di$\mathrm{BAC}_{4}(3)$ [32] after exposure to oxidized Crp4 and r-Crp4. r-Crp4 exhibited significantly greater depolarization than did oxidized Crp4 in 3 of the 3 commensal bacteria tested (fig. 7). On the other hand, the depolarization activities of oxidized Crp4 and r-Crp4 were not significantly different against 2 of 3 species of noncommensal bacteria (fig. 7). Both oxidized Crp4 and r-Crp4 showed a remarkable depolarization against $K$. oxytoca (fig. 7).

\section{Digestion of $r$-Crp4 by MMP-7 Resulted in the}

Attenuation of Its Bactericidal Activity

Crps are processed and activated by the processing enzyme, i.e. MMP-7, in vivo in Paneth cell granules. It has been reported that the disulfide bonds of Crp4 are essential for proteolytic resistance to MMP-7 due to the use of the disulfide bond-null mutant of Crp4 [25]. Therefore, to elucidate the susceptibility of r-Crp4 to MMP-7, an assay for proteolytic degradation was conducted. Oxidized Crp4 and r-Crp4 were incubated with or without MMP-7 and then applied to Tris-Tricine SDS-PAGE. r-Crp4 was digested by MMP-7 into smaller fragments, whereas oxidized Crp4 showed complete resistance to MMP-7 (fig. 8a). N-terminal peptide sequencing of the peptide fragment that emerged in the Tris-Tricine SDS-PAGE revealed that the N-terminus was YCRKG. MALDI-TOF MS showed that the peptide fragment had a molecular weight of 2,480 Da. These results indicated that MMP-7 cleaved r-Crp4 at Cys ${ }^{4} \downarrow \mathrm{Tyr}^{5}$ and $\mathrm{Phe}^{25} \downarrow \mathrm{Leu}^{26}$ as shown in figure $8 \mathrm{~b}$. Although the peptide fragment appeared to have a molecular weight of $\sim 3.5 \mathrm{kDa}$ in figure $8 \mathrm{a}$, because the fragment comprises cationic amino acids at high rates (theoretical $\mathrm{pI}=10.3$ ), it might have had a smaller migration in Tris-Tricine SDS-PAGE than that expected based on the molecular weight determined by MALDI-TOF MS. The observed cleavage sites correspond to the previous cleavage of reduced, alkylated proCrp4 by MMP-7 at the same sites [9]. Furthermore, the digested peptide fragments were assayed for bactericidal peptide activity to test for biological effects of the degradation. Previously, it was shown that MMP-7 itself had no effect on bactericidal activity $[7,25]$. The bactericidal activities of r-Crp4 against commensal and noncommensal bacteria, except for L. acidophilus, significantly decreased when $\mathrm{r}$-Crp4 was digested by MMP-7 (table 1). In contrast, the faint bactericidal activity of digested r-Crp4 
Table 1. Bactericidal activities of r-Crp4 and MMP-7-digested r-Crp4

\begin{tabular}{lrc}
\hline Bacteria & $\begin{array}{l}\text { Survival rates }{ }^{\mathrm{a}}(\%) \text { of } \\
\text { bacteria exposed to }\end{array}$ \\
\cline { 2 - 3 } & r-Crp4 & $\begin{array}{l}\text { MMP-7- } \\
\text { digested } \\
\text { r-Crp4 }\end{array}$ \\
\hline S. enterica serovar Typhimurium & \multicolumn{2}{c}{$\begin{array}{c}\text { r-4 } \\
\text { S. enterica serovar Typhimurium phoP- }\end{array}$} \\
E. coli ML35 & $3.2 \pm 2.7$ & $96.3 \pm 9.9^{\mathrm{b}}$ \\
S. aureus & $0.0 \pm 0.0$ & $118.1 \pm 10.9^{\mathrm{b}}$ \\
B. bifidum & $0.0 \pm 0.0$ & $49.5 \pm 13.4^{\mathrm{b}}$ \\
B. longum & $13.1 \pm 7.3$ & $96.4 \pm 10.4^{\mathrm{b}}$ \\
L. casei & $0.2 \pm 0.2$ & $87.3 \pm 17.0^{\mathrm{b}}$ \\
L. acidophilus & $28.8 \pm 7.3$ & $72.4 \pm 10.3^{\mathrm{b}}$ \\
B. thetaiotaomicron & $53.9 \pm 6.3$ & $64.8 \pm 0.9^{\mathrm{c}}$ \\
B. vulgatus & $33.9 \pm 5.7$ & $86.5 \pm 9.5^{\mathrm{b}}$ \\
\hline
\end{tabular}

${ }^{a}$ Values are means \pm SE for the survival rates of bacteria exposed to r-Crp4 (1.35 $\mu \mathrm{M})$ and MMP-7-digested r-Crp4 (1.35 $\mu \mathrm{M})$. $\mathrm{n}=6$ for $\mathrm{r}$-Crp $4 ; \mathrm{n}=3$ for MMP-7-digested $\mathrm{r}$-Crp4 .

${ }^{b}$ Value is significantly different $(\mathrm{p}<0.01)$ compared to that of bacteria exposed to r-Crp 4 as calculated by Student's t test.

${ }^{\mathrm{c}}$ Not significant.

against $L$. acidophilus remained (table 1). Thus, the degradation of $\mathrm{r}-\mathrm{Crp} 4$ by MMP-7 attenuated its bactericidal activity against most of the bacteria tested.

\section{Discussion}

In this study, the bactericidal activities of oxidized Crp4 against commensal and noncommensal bacteria were analyzed. Consistent with the results of previous studies [18, 25], oxidized Crp4 showed potent bactericidal activities against noncommensal bacteria. In contrast, oxidized Crp4 had only minimal or no bactericidal activity against commensal bacteria, though it did show dosedependent activity against $B$. longum, B. vulgatus, E. faecalis, and $E$. faecium. These results demonstrate that oxidized Crp4 has a more selective bactericidal activity against small intestinal bacteria. Thus, it is suggested that Crp4 plays a role in the regulation of intestinal microbiota by killing certain noncommensal species while retaining the viability of certain commensal bacteria. This concept was supported by the result that oxidized Crp4 selectively killed $S$. enterica serovar Typhimurium but showed no bactericidal activity against 2 species of com- mensal bacteria, i.e. L. casei and B. thetaiotaomicron, when a mixture of bacterial species was exposed to oxidized Crp4. Oxidized Crp1 also showed selective bactericidal activities, suggesting that other members of Crps contribute to maintaining intestinal microbiota by selectively killing noncommensal bacteria. A recent study of intestinal microbiota in MMP-7-deficient mouse types that lacked the active form of Crps showed that a significantly higher percentage of Firmicutes and a significantly lower percentage of Bacteroides were detected in the small bowel of MMP-7-deficient mice compared to wildtype mice [33]. Since the total bacterial numbers in both types of mouse were not changed, it appears that Crps regulated the composition of the intestinal bacteria [33], consistent with our results that demonstrate the selective bactericidal activity of Crp4.

Some commensal bacteria showed a resistance to oxidized Crp4. To date, various bacterial resistance mechanisms against AMPs have been reported [34]. These include the proteolytic degradation of AMPs by microbial proteases [35], the binding of secreted bacterial proteins to AMPs to prevent AMPs from accessing the bacterial plasma membrane [36], the extrusion of AMPs from the bacterial cell by a multiple drug resistance exporter [37], and the modification of the bacterial cell membrane to reduce the net anionic charge, resulting in an attenuation of the affinity of AMPs to the surface membrane [38, 39]. A wide variety of microbicidal mechanisms, such as the permeabilization of bacterial cell membranes and the inhibition of DNA or protein synthesis, are presented for various AMPs [40]. In the case of Crp4, it has been shown that Crp4 permeabilized the phospholipid bilayer and that the activity was dependent on the membrane composition [41]. However, the precise bactericidal mechanisms of native Crp4 have yet to be fully elucidated and may be bacteria dependent. Therefore, the bacterial resistance mechanisms against Crp4 may also be varied and bacteria dependent. We detected a depolarization of the membrane potential in some noncommensal bacteria by Crp4. Further investigation of the bacterial factors that affect bactericidal activity is needed to uncover the bacterial resistance mechanisms against Crp4.

In this study, Bifidobacterium sp., Lactobacillus sp., Bacteroides sp., and Enterococcus sp. were used as types of small intestinal commensal bacteria. These bacteria have been reported to colonize mouse small intestine where Crp4 is present [42, 43]. Oxidized Crp4 did not show bactericidal activities against $B$. bifidum, Lactobacillus sp., $B$. thetaiotaomicron, or B. fragilis, while it did kill B. longum, B. vulgatus, and Enterococcus sp. B. bifi- 
dum comprises the major portion of the intestinal microbiota in breast-fed infants [27]. B. longum is mainly found in adult intestine [28], and L. acidophilus, L. casei, L. johnsonii, B. thetaiotaomicron, B. fragilis, and B. vulgatus colonize the intestinal tract in both infants and adults [29, 30]. Enterococcus sp. are commensal bacteria that can cause opportunistic infection. It is speculated that the bacteria-dependent bactericidal activities of oxidized Crp4 against commensal bacteria reflect an in vivo regulatory role of Crp4 in tuning microbial homeostasis.

The bactericidal activities of $\mathrm{r}$-Crp4 which contained no disulfide bond against small intestinal bacteria were analyzed. $\mathrm{r}$-Crp 4 had significantly greater bactericidal activities against $B$. bifidum, B. breve, L. casei, L. johnso$n i i, B$. fragilis, B. ovatus, and B. thetaiotaomicron compared to oxidized Crp4. This result shows that the bactericidal activities of Crp4 against certain commensal bacteria were regulated by a function of its disulfide bonds, indicating a novel role of the conserved disulfide bonds of Crp4 in controlling bactericidal activities. r-Crp4 showed significantly greater depolarization activity than did oxidized Crp4 against the 3 commensal bacterial species tested. These results suggest that a disruption of membrane integration may account for part of the potent bactericidal activities of r-Crp4 relative to oxidized Crp4, especially with respect to certain commensals. Meanwhile, r-Crp4 and oxidized Crp4 showed equivalent bactericidal activities against 11 of 11 noncommensal bacteria and against 5 of 12 commensal bacteria. These results demonstrate that the bactericidal activities of Crp4 against these bacteria have no relationship with the presence of disulfide bonds. Previously, Maemoto et al. [25] showed that the bactericidal activity of Crp4 against noncommensal bacteria was independent of its disulfide array by using a disulfide-null mutant of Crp4. Our results show that the reduction of disulfide bonds has an effect against noncommensal bacteria compatible with the previous study. Thus, the regulatory effect of disulfide bonds depends on the bacterial species. r-Crpl also showed the bacteria-dependent regulatory effects of disulfide bonds. It is speculated that this property of disulfide bonds may be applicable to other Crps. Native Crp4 consists of a triple-stranded antiparallel $\beta$-sheet [44], whereas the NMR spectroscopy of Cys-to-Ala-substituted Crp4 indicates the mutant peptide is disordered [25]. According to these structural data, the r-Crp4 used in this study may also have a random coil structure. In that case, it is suggested that the flexibility of $\mathrm{r}-\mathrm{Crp} 4$ makes it bactericidal against the B. bifidum, L. casei, L. johnsonii, B. thetaiotaomicron, and $B$. fragilis that oxidized Crp4 did not kill completely.
In vitro degradation assays revealed that $\mathrm{r}$-Crp4 was digested by MMP-7. Further, the bactericidal activities of r-Crp4 against small intestinal microbiota, except for L. acidophilus, were attenuated by this digestion. If r-Crp4 were to be secreted into the lumen of the small intestine, r-Crp4 would kill both noncommensal and commensal bacteria, resulting in the perturbation of small intestinal microbial homeostasis. Thus, it is considered that degradation and inactivation of $\mathrm{r}$-Crp4 before secretion would prevent this perturbation. This suggests that the host has a management mechanism to avoid the release of aberrant Crp4 that is disadvantageous to the host.

Previously, it was reported that the proform of human Paneth cell $\alpha$-defensin, i.e. HD5, was reduced in some patients with Crohn's disease [45]. The reduced pro-HD5 was degraded by trypsin, a processing enzyme of HD5, in vivo. This resulted in a diminished production of mature HD5 [45]. If Crp4 were to be reduced in vivo, the degradation of Crp4 by MMP-7 would lead to the dysfunction of innate immunity. Therefore, these results indicate that the protease resistance of $\alpha$-defensins due to the disulfide bonds may contribute to the maintenance of intestinal innate immunity as well as the pathology of diseases such as inflammatory bowel disease.

\section{Acknowledgements}

We are grateful to Prof. A.J. Ouellette (University of Southern California) for helpful discussions. This work was supported by a Grant-in-Aid for Frontier Technology Research from the Northern Advancement Center for Science and Technology (NOASTEC) of Japan (T.A.), a Grant-in-Aid for Knowledge Cluster Phase II, Sapporo Bio-S, and a Grant-in-Aid for Scientific Research on Priority Areas from the Ministry of Education, Culture, Sports, Science, and Technology of Japan (T.A., N.S. and K.N.). This work was also partially supported by a Grant-in-Aid for Young Scientists (B) (N.S.) and by a Grant-in-Aid for Scientific Research (C) (K.N.) from the Ministry of Education, Culture, Sports, Science and Technology of Japan.

References

J Innate Immun 2011;3:315-326
Zasloff M: Antimicrobial peptides of multicellular organisms. Nature 2002;415:389-395.

$\checkmark 2$ Ganz T: Defensins: antimicrobial peptides of innate immunity. Nat Rev Immunol 2003;3: $710-720$.

3 Selsted ME, Ouellette AJ: Mammalian defensins in the antimicrobial immune response. Nat Immunol 2005;6:551-557.

-4 Ayabe T, Satchell DP, Wilson CL, Parks WC, Selsted ME, Ouellette AJ: Secretion of microbicidal $\alpha$-defensins by intestinal Paneth cells in response to bacteria. Nat Immunol 2000; 1:113-118. 
5 Ayabe T, Ashida T, Kohgo Y, Kono T: The role of Paneth cells and their antimicrobial peptides in innate host defense. Trends Microbiol 2004;12:394-398.

-6 Salzman NH, Underwood MA, Bevins CL: Paneth cells, defensins, and the commensal microbiota: a hypothesis on intimate interplay at the intestinal mucosa. Semin Immunol 2007;19:70-83.

7 Wilson CL, Ouellette AJ, Satchell DP, Ayabe T, López-Boado YS, Stratman JL, Hultgren SJ, Matrisian LM, Parks WC: Regulation of intestinal $\alpha$-defensin activation by the metalloproteinase matrilysin in innate host defense. Science 1999;286:113-117.

$\checkmark 8$ Ayabe T, Satchell DP, Pesendorfer P, Tanabe H, Wilson CL, Hagen SJ, Ouellette AJ: Activation of Paneth cell $\alpha$-defensins in mouse small intestine. J Biol Chem 2002;277:52195228.

9 Shirafuji Y, Tanabe H, Satchell DP, Henschen-Edman A, Wilson CL, Ouellette AJ: Structural determinants of procryptdin recognition and cleavage by matrix metalloproteinase-7. J Biol Chem 2003;278:7910-7919.

$\checkmark 10$ Dethlefsen L, McFall-Ngai M, Relman DA: An ecological and evolutionary perspective on human-microbe mutualism and disease. Nature 2007;449:811-818.

- 11 Koropatnick TA, Engle JT, Apicella MA, Stabb EV, Goldman WE, McFall-Ngai MJ: Microbial factor-mediated development in a host-bacterial mutualism. Science 2004;306: 1186-1188.

\12 Turnbaugh PJ, Ley RE, Mahowald MA, Magrini V, Mardis ER, Gordon JI: An obesity-associated gut microbiome with increased capacity for energy harvest. Nature 2006; 444:1027-1031.

13 Mazmanian SK, Liu CH, Tzianabos AO, Kasper DL: An immunomodulatory molecule of symbiotic bacteria directs maturation of the host immune system. Cell 2005;122: 107-118.

14 Artis D: Epithelial-cell recognition of commensal bacteria and maintenance of immune homeostasis in the gut. Nat Rev Immunol 2008;8:411-420.

15 Mazmanian SK, Round JL, Kasper DL: A microbial symbiosis factor prevents intestinal inflammatory disease. Nature 2008;453: 620-625.

16 Karin M, Lawrence T, Nizet V: Innate immunity gone awry: linking microbial infections to chronic inflammation and cancer. Cell 2006;124:823-835.

17 Ouellette AJ, Lualdi JC: A novel mouse gene family coding for cationic, cysteine-rich peptides: regulation in small intestine and cells of myeloid origin. J Biol Chem 1990;265: 9831-9837.

18 Ouellette AJ, Hsieh MM, Nosek MT, CanoGauci DF, Huttner KM, Buick RN, Selsted ME: Mouse Paneth cell defensins: primary structures and antibacterial activities of numerous cryptdin isoforms. Infect Immun 1994;62:5040-5047.
9 Hornef MW, Putsep K, Karlsson J, Refai E, Andersson M: Increased diversity of intestinal antimicrobial peptides by covalent dimer formation. Nat Immunol 2004;5:836-843.

20 Patil A, Hughes AL, Zhang G: Rapid evolution and diversification of mammalian $\alpha$-defensins as revealed by comparative analysis of rodent and primate genes. Physiol Genomics 2004;20:1-11.

21 Mastroianni JR, Ouellette AJ: $\alpha$-Defensins in enteric innate immunity: functional Paneth cell $\alpha$-defensins in mouse colonic lumen. J Biol Chem 2009;284:27848-27856.

22 Ouellette AJ, Darmoul D, Tran D, Huttner KM, Yuan J, Selsted ME: Peptide localization and gene structure of cryptdin 4 , a differentially expressed mouse Paneth cell $\alpha$-defensin. Infect Immun 1999;67:6643-6651.

23 Satchell DP, Sheynis T, Kolusheva S, Cummings J, Vanderlick TK, Jelinek R, Selsted ME, Ouellette AJ: Quantitative interactions between cryptdin- 4 amino terminal variants and membranes. Peptides 2003;24:17951805.

-24 Tanabe H, Qu X, Weeks CS, Cummings JE, Kolusheva S, Walsh KB, Jelinek R, Vanderlick TK, Selsted ME, Ouellette AJ: Structure-activity determinants in Paneth cell $\alpha$-defensins: loss-of-function in mouse cryptdin- 4 by charge-reversal at arginine residue positions. J Biol Chem 2004;279:11976-11983.

25 Maemoto A, Qu X, Rosengren KJ, Tanabe H, Henschen-Edman A, Craik DJ, Ouellette AJ: Functional analysis of the $\alpha$-defensin disulfide array in mouse cryptdin-4. J Biol Chem 2004;279:44188-44196.

26 Selsted ME, Brown DM, DeLange RJ, Lehrer RI: Primary structures of MCP-1 and MCP2 , natural peptide antibiotics of rabbit lung macrophages. J Biol Chem 1983;258:1448514489.

27 Weiss JE, Rettger LF: Lactobacillus bifidus. J Bacteriol 1934;28:501-521.

28 Reuter G: Designation of type strains for Bifidobacterium species. Int J Syst Bacteriol 1971;21:273-275.

29 Dellaglio F, Bottazzi V, Vescovo M: Deoxyribonucleic acid homology among Lactobacillus species of the subgenus Streptobacterium Orla-Jensen. Int J Syst Bacteriol 1975;25:160172.

-30 Hansen PA, Mocquot G: Lactobacillus acidophilus (Moro) comb. nov. Int J Syst Bacteriol 1970;20:325-327.

- 31 Fields PI, Groisman EA, Heffron F: A Salmonella locus that controls resistance to microbicidal proteins from phagocytic cells. Science 1989;243:1059-1062.

32 Nuding S, Fellermann K, Wehkamp J, Mueller HA, Stange EF: A flow cytometric assay to monitor antimicrobial activity of defensins and cationic tissue extracts. J Microbiol Methods 2006;65:335-345.

33 Salzman NH, Hung K, Haribhai D, Chu H, Karlsson-Sjoberg J, Amir E, Teggatz P, Barman M, Hayward M, Eastwood D, Stoel M, Zhou Y, Sodergren E, Weinstock GM, Bevins
CL, Williams CB, Bos NA: Enteric defensins are essential regulators of intestinal microbial ecology. Nat Immunol 2010;11:76-83.

34 Peschel A, Sahl HG: The co-evolution of host cationic antimicrobial peptides and microbial resistance. Nat Rev Microbiol 2006;4: 529-536.

35 Guina T, Yi EC, Wang H, Hackett M, Miller SI: A PhoP-regulated outer membrane protease of Salmonella enterica serovar Typhimurium promotes resistance to alphahelical antimicrobial peptides. J Bacteriol 2000;182:4077-4086.

36 Jin T, Bokarewa M, Foster T, Mitchell J, Higgins J, Tarkowski A: Staphylococcus aureus resists human defensins by production of staphylokinase, a novel bacterial evasion mechanism. J Immunol 2004;172:1169-1176.

- 37 Shafer WM, Qu X, Waring AJ, Lehrer RI: Modulation of Neisseria gonorrhoeae susceptibility to vertebrate antibacterial peptides due to a member of the resistance/nodulation/division efflux pump family. Proc Natl Acad Sci USA 1998;95:1829-1833.

38 Guo L, Lim KB, Poduje CM, Daniel M, Gunn JS, Hackett M, Miller SI: Lipid A acylation and bacterial resistance against vertebrate antimicrobial peptides. Cell 1998;95:189198.

39 Peschel A, Jack RW, Otto M, Collins LV, Staubitz P, Nicholson G, Kalbacher H, Nieuwenhuizen WF, Jung G, Tarkowski A, van Kessel KP, van Strijp JA: Staphylococcus aureus resistance to human defensins and evasion of neutrophil killing via the novel virulence factor $\mathrm{MprF}$ is based on modification of membrane lipids with l-lysine. J Exp Med 2001;193:1067-1076.

40 Brogden KA: Antimicrobial peptides: pore formers or metabolic inhibitors in bacteria? Nat Rev Microbiol 2005;3:238-250.

41 Hadjicharalambous C, Sheynis T, Jelinek R, Shanahan MT, Ouellette AJ, Gizeli E: Mechanisms of $\alpha$-defensin bactericidal action: comparative membrane disruption by cryptdin- 4 and its disulfide-null analogue. Biochemistry 2008;47:12626-12634.

42 Wagner RD, Warner T, Roberts L, Farmer J, Balish E: Colonization of congenitally immunodeficient mice with probiotic bacteria. Infect Immun 1997;65:3345-3351.

43 Ménard O, Butel M, Gaboriau-Routhiau V, Waligora-Dupriet A: Gnotobiotic mouse immune response induced by Bifidobacterium sp. strains isolated from infants. Appl Environ Microbiol 2008;74:660-666.

-44 Jing WG, Hunter HN, Tanabe H, Ouellette AJ, Vogel HJ: Solution structure of cryptdin-4, a mouse Paneth cell $\alpha$-defensin. Biochemistry 2004;43:15759-15766.

-45 Tanabe H, Ayabe T, Maemoto A, Ishikawa C, Inaba Y, Sato R, Moriichi K, Okamoto K, Watari J, Kono T, Ashida T, Kohgo Y: Denatured human $\alpha$-defensin attenuates the bactericidal activity and the stability against enzymatic digestion. Biochem Biophys Res Commun 2007;358:349-355. 